\title{
TFIID can be rate limiting in vivo for TATA-containing, but not TATA-lacking, RNA polymerase II promoters
}

\author{
John Colgan and James L. Manley \\ Department of Biological Sciences, Columbia University, New York, New York 10027 USA
}

\begin{abstract}
We have studied the effect of exogenous expression of the basal transcription factor TFIID on the activities of several different TATA-containing and TATA-lacking promoters. Overexpression of TFIID from a transfected plasmid in Drosophila Schneider cells resulted in substantial concentration-dependent increases in expression from a cotransfected minimal TATA-containing promoter. Overexpression of TFIID activated expression from all TATA-containing promoters tested, with the maximum level of activation being inversely proportional to the strength of the promoter. In contrast, expression from TATA-less promoters was not enhanced, and could in fact be reduced, by increased expression of TFIID. Consistent with these findings overexpression of TFIID had opposite effects on Sp1-mediated activation observed from minimal synthetic promoters consisting of Sp1-binding sites and either a TATA box or initiator element. We discuss the significance of these results in terms of the role of TFIID in the initiation of transcription and as a possible regulatory target for expression from TATA-containing promoters, as well as the role TFIID may play in expression from TATA-less promoters.
\end{abstract}

[Key Words: TFIID-promoter interactions; transcriptional regulation; transcription initiation; Drosophila]

Received August 5, 1991; revised version accepted December 9, 1991.

Initiation of transcription by RNA polymerase II involves interactions between the polymerase, several essential transcription factors, and promoter DNA. To understand this critical process, the characteristics and functions of the factors necessary for the initiation reaction must be determined. Through fractionation of nuclear extracts from mammalian cells, six factors, designated TFIIA, B, D, E, F, and $G$, have been identified as being required in addition to the multisubunit RNA polymerase for initiation from a minimal class II promoter (Reinberg and Roeder 1987; Reinberg et al. 1987; Nakajima et al. 1988; Flores et al. 1990; Ohkuma et al. 1990; Sumimoto et al. 1990; for review, see Mermelstein et al. 1989; Sawadogo and Sentenac 1990|. Counterparts to some of these essential factors have been shown to exist in Drosophila (e.g., Wampler et al. 1990) as well as in yeast (Buratowski et al. 1988; Cavallini et al. 1988; Hahn et al. 1989c), and the functional properties required for interactions between these essential components appear to have been well conserved during evolution, as certain factors can function in place of their counterparts in vitro in heterologous reconstituted systems (Buratowski et al. 1988; Cavallini et al. 1988; Hahn et al. 1989c; Flanagan et al. 1990; Wampler et al. 1990).

The promoters of genes transcribed by RNA polymerase II commonly contain a conserved sequence element known as the TATA box (Breathnach and Chambon 1981), although a subset, referred to as TATA-less promoters, lack sequences that bear any significant homology to this element (for review, see Dynan 1986). Kinetic and template commitment studies using TATA-containing promoters have indicated that two factors, TFIIA and TFIID, act at an early step in the initiation pathway (Davison et al. 1983; Fire et al. 1984; Reinberg et al. 1987). Analysis of factor-template interactions in vitro have identified TFIID as the basal factor responsible for TATA box binding (Davison et al. 1983; Nakajima et al. 1988), which is required to promote transcription complex formation (Van Dyke et al. 1988; Buratowski et al. 1989) as well as to prevent repression of transcription by nucleosome assembly (Workman and Roeder 1987). Studies defining the events leading to initiation from TATA-less promoters have yet to be reported, but it appears that TFIID and the other essential factors are required for this process as well (Pugh and Tjian 1990; Smale et al. 1990; Carcamo et al. 1991).

The cloning of the TFIID gene from yeast /Cavallini et al. 1989; Eisenmann et al. 1989; Hahn et al. 1989b; Horikoshi et al. 1989; Schmidt et al. 1989) led to the isolation of cDNA clones encoding TFIID from a host of organisms, including plant (Gasch et al. 1990), human (Hoffmann et al. 1990; Kao et al. 1990; Peterson et al. 1990), 
and Drosophila (Hoey et al. 1990; Muhich et al. 1990). Comparison of the primary amino sequence derived from TFIID cDNAs from these and other organisms has revealed that the TFIID protein is bipartite, composed of a highly conserved carboxy-terminal domain of $\sim 180$ amino acid residues and an amino-terminal region that is variable with respect to length and amino acid content. Analysis of recombinant TFIID proteins has shown that only the carboxy-terminal core is required for TATA box binding and support of basal transcription in vitro, whereas the amino-terminal region has been implicated in mediating the response of TFIID to certain upstream activator proteins (Hoey et al. 1990; Horikoshi et al. 1990; Peterson et al. 1990; Pugh and Tjian 1990). Gene replacement studies done in yeast have shown that the carboxy-terminal domain alone is sufficient for growth and have also revealed that species-specific differences exist within this region, despite its high degree of conservation (Cormack et al. 1991; Gill and Tjian 1991; Reddy and Hahn 1991).

TFIID has been suggested to be a likely target for regulation by transcriptional activator proteins (for review, see Mitchell and Tjian 1989; Johnson and McKnight 1990; Ptashne and Gann 1990) in large part because it functions as the initiator of transcription complex formation. A number of in vitro studies have provided evidence consistent with this idea, as several factors have been shown to influence the DNA-binding characteristics of TFIID (Sawadogo and Roeder 1985; Horikoshi et al. 1988a,b; Sawadogo 1988; Meisternerst and Roeder 1991), while others appear to affect the rate and/or stability of TATA box binding under certain conditions, including during competition with chromatin assembly (Abamyr et al. 1988; Workman et al. 1988, 1990, 1991; Meisternerst et al. 1990). The functional contacts, if any, that occur between activator proteins and TFIID have not yet been identified, but two viral activator proteins have been shown to bind to TFIID directly (Stringer et al. 1990; Horikoshi et al. 1991; Lee et al. 1991). It also appears that at least some upstream activator proteins can influence the activity of TFIID through a class of intermediary molecules known as coactivators or adaptors (Berger et al. 1990; Kelleher et al. 1990; Martin et al. 1990; Pugh and Tjian 1990; for review, see Lewin 1990), examples of which have been partially purified from mammalian and Drosophila nuclear extracts (Dynlacht et al. 1991; Meisternerst et al. 1991).

The idea that TFIID-promoter interactions are promoted by certain activator proteins suggests that these factors may serve to recruit TFIID to the template which, in turn, allows for higher promoter activity. The importance of such interactions for transcriptional regulation would be heightened if TFIID were present in limiting quantities in vivo. Here, we present evidence that TFIID is maintained at a lower effective concentration relative to the other essential transcription factors within Drosophila cells and that TFIID can be rate limiting in vivo for expression from TATA-containing promoters. In contrast, we also show that TFIID is not limiting for expression from TATA-lacking promoters but appears to associate with another factor that is limiting for such promoters.

\section{Results \\ Wild-type and mutant TFIID protein can be produced in transfected cells}

Before embarking on an analysis of the possible effects of increased TFIID expression on promoter activity, we wished to verify that wild-type and mutant TFIID proteins could be transiently overexpressed in transfected cells. To this end, a cDNA encoding the Drosophila TFIID protein (kindly provided by $T$. Hoey and R. Tiian) was fused at the $5^{\prime}$ end of the coding region to a fragment encoding an 11-amino-acid sequence from the influenza virus HAl (Wilson et al. 1984; Field et al. 1988) and then inserted into an expression vector containing the Drosophila actin $5 \mathrm{C}$ distal promoter. The resulting plasmid (Act flu-TFIID) was introduced into Drosophila Schneider cells by transient cotransfection as described previously (Han et al. 1989). Western blot analysis of wholecell extracts from cells transfected with Act flu-TFIID using a monoclonal antibody that recognizes the flu epitope (Niman et al. 1983) revealed a protein of $\sim 38 \mathrm{kD}$ (Fig. 1A), a molecular mass identical essentially to that of the Drosophila TFIID protein (Hoey et al. 1990). When extracts prepared from cells transfected with increasing concentrations of Act flu-TFIID were analyzed, the amount of flu-TFIID protein detected increased in a manner directly proportional to the amount of expression vector (Fig. 1A).

We also analyzed the expression of two mutant forms of TFIID. Western blotting of extracts from cells transfected with Act flu-TFID $\Delta$ Nde (Fig. 1A), which should express the evolutionarily conserved carboxy-terminal core of TFIID joined to the flu epitope, and Act fluTFIID $\Delta \mathrm{Bgl}$ (Fig. 1A\}, which encodes a flu-TFIID fusion protein with 17 amino acids deleted from the carboxyl terminus, confirmed that both of these proteins were also expressed in transfected cells. The accumulation of these two mutant forms, however, was somewhat lower than observed for the full-length protein, suggesting that the mutant proteins may be somewhat unstable. As with Act flu-TFIID, the amount of each mutant protein detected was dependent on the amount of TFIID expression plasmid transfected (Fig. 1A). Therefore, we conclude that both wild-type and mutant TFIID proteins can be stably expressed from a plasmid in Drosophila Schneider cells. Furthermore, the amount of these proteins produced can be modulated by altering the amount of expression plasmid.

\section{Increased expression of TFIID activates a minimal TATA promoter}

The above experiments suggested that it would be feasible to determine whether increasing the level of TFIID could influence the expression of a cotransfected re- 
A

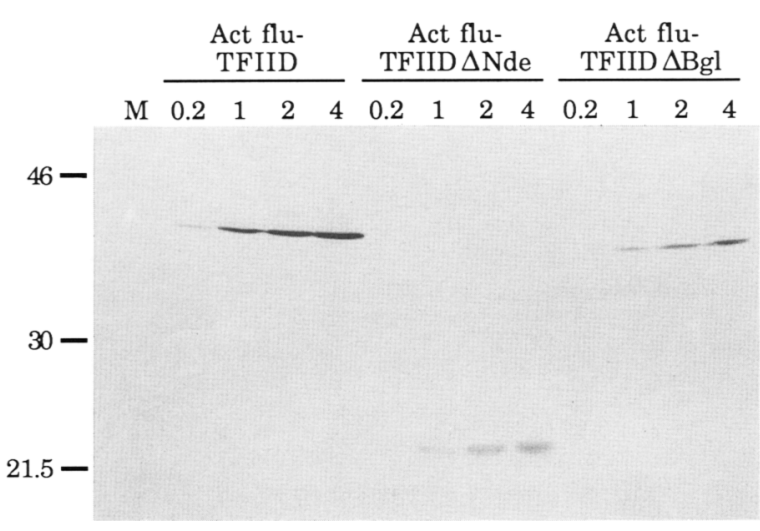

B

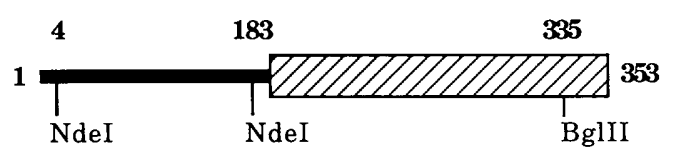

Figure 1. Expression of wild-type and mutant TFIID proteins. (A) Western blot analysis of extracts from cells transfected with $4 \mu \mathrm{g}$ of Act-PPA (M) or increasing concentrations of the indicated flu-TFIID expression plasmids as described in Materials and methods. The predicted sizes of the expressed proteins are $39 \mathrm{kD}$ for Act flu-TFIID, $22 \mathrm{kD}$ for Act flu-TFIID $\Delta$ Nde, and 38 $\mathrm{kD}$ for Act-TFIID $\Delta \mathrm{Bgl}$. The mobility of molecular mass marker proteins is indicated in kilodaltons $($ left $t) .(B)$ Schematic depicting the structure of TFIID and showing the location of restriction sites used to create expression vectors encoding wild-type and mutant forms of TFIID fused to the flu epitope as decribed in Materials and methods. The filled box represents the aminoterminal domain; the hatched box represents the conserved carboxy-terminal core domain of TFIID.

porter gene. We chose to analyze initially the effect of increasing the concentration of TFIID on transcription from a very weak minimal promoter. For this, a reporter plasmid containing only the adenovirus E1b-TATA se- quence (a gift of K. Martin and M. Green) inserted up stream of the bacterial chloramphenicol acetyltransferase (CAT) gene was cotransfected in the presence or absence of increasing concentrations of Act flu-TFIID. Alone, the level of CAT activity detected was barely above background. However, in the presence of Act fluTFIID, expression was strikingly enhanced, reaching a maximum increase of $\sim 135$-fold at the highest amount of expression vector tested (Fig. 2A). That this enhanced expression was dependent on the TATA element was verified by deletion of the TATA sequence from the reporter plasmid, which not only eliminated the weak basal CAT activity but also abolished completely the response of the plasmid to any amount of Act flu-TFIID tested (Fig. 2A).

Plasmids producing the truncated forms of TFIID were also tested for their ability to increase CAT gene expression from the Elb-TATA element. Cotransfection of Act flu-TFIID $\Delta$ Nde with Elb-TATA CAT also showed concentration-dependent activation of CAT activity (Fig. 2B). Somewhat lower levels of activation were observed with Act flu-TFIID $\Delta$ Nde (50-fold vs. 135-fold), most likely owing, at least in part, to the reduced accumulation of the truncated protein in transfected cells relative to the full-length protein. This finding is consistent with previous experiments showing that only the carboxy-terminal core of TFIID is required to support basal transcription (Hoey et al. 1990; Horikoshi et al. 1990; Peterson et al. 1990; Cormack et al. 1991; Gill and Tjian 1991; Reddy and Hahn 1991). The mutant form of TFIID lacking the carboxy-terminal 18 amino acids (Act fluTFIID $\Delta \mathrm{Bgl}$ ) failed to activate CAT expression from ElbTATA CAT at any concentration tested (Fig. 2B). This suggests that the exogenously expressed TFIID must bind DNA to activate CAT expression, as previous studies using the cloned yeast gene demonstrated that the entire conserved region of TFIID is required for both TATA box binding and initiation of transcription in vitro (Horikoshi et al. 1990).

These experiments establish that increased expression of functional TFIID molecules can enhance significantly
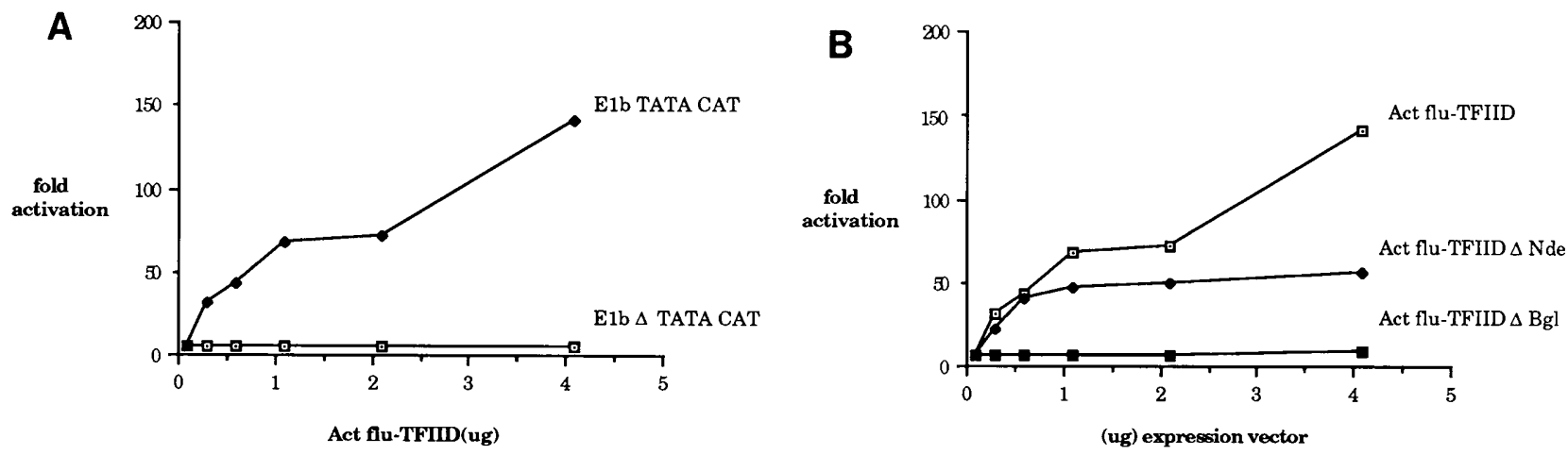

Figure 2. TFIID can activate a minimal TATA box promoter. $(A)$ Plot of relative CAT values obtained from cotransfections containing the E1b-TATA CAT or the E1b $\Delta$-TATA CAT reporter plasmid with increasing concentrations of the TFIID expression plasmid. (B) Plot of relative CAT values obtained from cotransfections containing the E1b-TATA CAT reporter with increasing concentrations of the indicated TFIID expression plasmid. The total amount of expression vector in each sample was adjusted to $4 \mu \mathrm{g}$ by the addition of Act-PPA as needed (see Materials and methods). 
transcription from a transfected reporter construct in a TATA box-dependent fashion. The results also indicate that TFIID is present in limiting quantities within the transfected cells because increased production of this protein allows an increase in the rate of transcription from a minimal TATA-containing promoter.

Relative activation of TATA-containing promoters by
increased TFIID is dependent on promoter strength

Expression from the Elb-TATA CAT plasmid was extremely weak in the absence of exogenous TFIID. To determine whether the "strength" of a promoter might influence its response to TFIID, a variety of different TATA-containing promoters were tested for activation by increased levels of TFIID. These experiments were performed with a TFIID expression vector that lacked the flu sequences (Act-TFIID), but preliminary experiments indicated that these sequences did not affect TFIID activity (results not shown). We first tested two very strong promoters. The $2.6-\mathrm{kb}$ actin $5 \mathrm{C}$ promoter, used to express TFIID from transfected plasmids owing to its high rate of transcription in Schneider cells, showed only slight increases in activity /approximately twofold) when fused to CAT and cotransfected with Act-TFIID (Table 1). The modest response of the actin $5 \mathrm{C}$ promoter to increased TFIID expression was not the result of some other limitation (e.g., exceeding the translational capacity of the transfected cells|, as significantly higher CAT activities (>2000 relative units; see Table 1) could be obtained from transfections that included increased concentrations of the actin $5 \mathrm{C}$ reporter plasmid (data not shown). A reporter construct containing a 137bp promoter fragment $[-47$ to +90 ; lacking heat shock factor (HSF)-binding sites] taken from the Drosophila $70 \mathrm{~K}$ heat shock gene (hsp 70; Ingolia et al. 1980), another relatively strong promoter, was also activated to a maximum of two- to threefold by the highest amount of ActTFIID tested (Table 1). Transcription from both promot-

Table 1. TFIID can increase TATA box promoter activity

\begin{tabular}{lccccc}
\hline $\begin{array}{l}\text { Act-TFIID } \\
(\mu \mathrm{g})\end{array}$ & $\begin{array}{l}\text { Actin } \\
\text { 5C }\end{array}$ & hsp70 & $\begin{array}{c}\text { hsp70 } \\
\Delta \text { Acc }\end{array}$ & Met & SV40 E \\
\hline- & 30.6 & 132 & 0.5 & 1.5 & 1.0 \\
0.2 & 39.6 & 156 & 3.1 & 10.6 & 15.1 \\
1.0 & 53.2 & 262 & 4.6 & 19.1 & 30.1 \\
4.0 & 64.3 & 280 & 6.6 & 16.3 & 28.0 \\
Max activation & $2.1 \times$ & $2.1 \times$ & $13.2 \times$ & $12.7 \times$ & $30.1 \times$ \\
\hline
\end{tabular}

Cotransfections with the indicated plasmids were performed as described in Materials and methods, except the amount of the Act CAT reporter plasmid used was only $0.05 \mu \mathrm{g}$. Values in the upper part are displayed as the ratio between CAT activity and the internal control ( $\beta$-gal activity) for each promoter in the presence of the indicated amount of Act-TFIID; the lower part gives the maximum fold activation observed in the presence of Act-TFIID relative to the promoter activity obtained in its absence. ers was stimulated by Act-TFIID in a concentration-dependent manner, indicating that increased TFIID expression was responsible for the observed activation.

Several weaker promoters gave rise to intermediate levels of activation. $h s p 70 \Delta \mathrm{Acc} C \mathrm{CAT}$, which contains a 43-bp promoter fragment from the $h s p 70$ gene $(-49$ to $-6)$, and thus the same TATA box and surrounding sequences found in $h s p 70 \mathrm{CAT}$, displayed a much lower basal level than hsp70 CAT (0.5 vs. 133; see Table 1). This difference is presumably the result of the presence of activator protein-binding sites in the $h s p 70$ CAT promoter that are absent in $h s p 70 \Delta A c c$ CAT (Parker and Topol 1984; Gilmour et al. 1989, 1990). Comparison of TFIID-mediated activation of $h s p 70$ and $h s p 70 \Delta \mathrm{Acc}$ (Table 1 ) indicates that the weaker promoter showed significantly higher relative activation levels, reaching a maximum of 13-fold induction over basal expression. Two other weakly expressed TATA-containing fragments, taken from the Drosophila metallothionein promoter (Bunch et al. 1988) and the SV40 early promoter (SV2 CAT; Gorman et al. 1982), also showed relatively strong activation $(\sim 15$-fold and 30-fold, respectively; see Table 1). These experiments indicate that an inverse relationship exists between promoter strength and relative activation by increased TFIID expression.

\section{Increasing levels of TFIID do not enhance basal expression from TATA-less promoters}

A plasmid expressing $\beta$-galactosidase from the copia long terminal repeat (LTR) (Mount and Rubin 1985), a TATA-less promoter, was used in the above experiments to monitor transfection efficiencies (Han et al. 1989). Somewhat unexpectedly, no variations in $\beta$-galactosidase ( $\beta$-gal) activity were observed between extracts from cells cotransfected with Act-TFIID and those cotransfected with the control expression vector, Act-PPA (Han et al. 1989; data not shown). Although these findings validated the use of this plasmid as an internal control, they also prompted us to determine the effect of increased TFIID expression on other TATA-less promoters.

We first tested a relatively strong TATA-less promoter fragment $(-38$ to +172$)$ from the segment polarity gene engrailed (en; Poole et al. 1985; Soeller et al. 1988), which gives rise to approximately the same CAT expression as the $h s p 70$ promoter described above (132 vs. 158). In contrast to the $h s p 70$ promoter, which was activated in a concentration-dependent fashion by cotransfection with Act-TFIID, the TATA-less en promoter showed essentially no response to the addition of any amount of TFIID expression vector (Table 2). This was not the result of the strength of the en promoter, as expression levels from a second reporter containing a smaller en promoter fragment ( +2 to +117 ; en $\Delta$ Hinc CAT) and another TATA-less promoter fragment, from the homeotic gene Ultrabithorax (Ubx; Hogness et al. 1985), were also not enhanced by TFIID despite their significantly decreased basal activities (158 vs. $\sim 7$; see Table 2 ). CAT expression from a copia LTR reporter plasmid, which by 
Table 2. TFIID does not enhance TATA-less promoter activity

\begin{tabular}{lcccc}
\hline $\begin{array}{l}\text { Act-TFII } \\
(\mu \mathrm{g})\end{array}$ & en & copia & Ubx & en $\Delta$ Hinc \\
\hline- & 148 & 12.3 & 7.5 & 7.1 \\
0.2 & 164 & 11.0 & 5.4 & 5.8 \\
1.0 & 150 & 7.7 & 5.5 & 6.2 \\
4.0 & 170 & 8.9 & 6.2 & 6.0 \\
Max repression & $1.0 \times$ & $0.6 \times$ & $0.7 \times$ & $0.8 \times$ \\
\hline
\end{tabular}

Cotransfections with the indicated plasmids were performed as described in Materials and methods. Values in the upper part are displayed as the ratio between CAT activity and the internal control ( $\beta$-gal activity) for each promoter in the presence of the indicated amount of Act-TFIID; the lower part gives the maximum fold repression observed in the presence of Act-TFIID relative to the promoter activity obtained in its absence.

itself was also relatively weak, likewise showed no activation when cotransfected with Act-TFIID. Slight decreases in expression from all three of these weaker promoters were reproducibly observed (Table 2). Therefore, in contrast to the behavior of all TATA-containing promoters tested, all of the TATA-less promoters used here showed no increases in expression upon cotransfection with Act-TFIID; this indicates that TFIID is not rate limiting for transcription from TATA-less promoters under conditions where it is for TATA-containing promoters.

Overexpression of TFIID has opposite effects on TATA-containing and TATA-lacking promoters during competition with excess TATA sequences

As described above, addition of exogenous TFIID allowed increased expression from all TATA-containing promoters tested, indicating that the endogenous TFIID was present in concentrations that limited their activity. In contrast, expression from TATA-less promoters was not enhanced by overexpression of TFIID, which suggests that TFIID is not involved in expression from promoters of this type or, alternatively, that TFID is not a limiting factor for TATA-less promoter activity. To test whether the endogenous TFIID is limiting for TATA-containing promoters and to determine whether TFIID is required for TATA-less promoter expression, we attempted to create competition for the endogenous TFIID. For this, a set of "competitor" plasmids was constructed by inserting either TATA-containing or TATA-less promoter fragments into a plasmid lacking a CAT gene. Each of these was then cotransfected with hsp 70 CAT or en CAT plasmids under conditions in which the competitor was present in a large molar excess (40-fold) over the reporter.

We initially tested a competitor plasmid containing the same promoter fragment as the TATA-containing reporter itself (GEM $h s p$ ) and found that expression from hsp70 CAT was reduced by a factor of $\sim 2.5$ (Table 3 ). This reduction proved to be largely TATA dependent, as deletion of the TATA element from the $h s p 70$ promoter fragment severely impaired its ability to serve as a competitor (data not shown). TATA-specific inhibition was also observed using the plasmid GEM Met-TATA, which bears a 33-bp TATA-containing fragment $\mid-41$ to $-8)$ from the metallothionein promoter. This competitor was able to reduce CAT expression from $h s p 70$ by a factor of $\sim 5$ (Table 3 ). In contrast, a plasmid containing a TATA-less promoter fragment from the en gene produced, at most, only slight reductions in expression from the $h s p 70$ reporter plasmid.

To determine whether the endogenous TFIID was actually the limiting factor being competed away in the above experiments, we tested whether expression from the TATA-containing reporter could be restored by adding Act-TFIID to transfection mixtures in place of ActPPA (see Materials and methods) during competition. This resulted in significantly increased activity from the hsp70 reporter during competition with GEM hsp or GEM Met-TATA (Table 3) competitor plasmids. Collectively, these experiments indicate that excess TATAcontaining promoter fragments can inhibit transcription from the $h s p 70$ reporter by competing for the endogenous TFIID and that increased expression of TFIID can counteract such inhibition.

As with the $h s p 70$ reporter, cotransfection of the competitor plasmids with the TATA-less en reporter plasmid brought about reductions in CAT activity. The TATAcontaining competitor GEM hsp gave rise to a modest inhibition of CAT expression (1.5-fold; see Table 3). Cotransfection with GEM Met-TATA or GEM en, which carries the same TATA-less promoter fragment as the reporter gene tested, also brought $\sim 1.5$-fold reduction in CAT expression. Although the effects on the en promoter were small, they have been observed consistently in multiple, independent experiments. In contrast to the results obtained with hsp $70 \mathrm{CAT}$, when Act-

Table 3. TFIID can restore activity from TATA-containing, but not TATA-less, promoters during competition

\begin{tabular}{llll}
\hline & & \multicolumn{2}{c}{ Expression vector } \\
\cline { 3 - 4 } Promoter & Competitor & ActpBR & Act-TFIID \\
\hline hsp70 (TATA) & GEM3 & 1.0 & 2.3 \\
& GEM $e n$ & 0.79 & 1.57 \\
& GEM $h s p$ & 0.40 & 1.02 \\
& GEM Met- & & \\
& TATA & 0.20 & 0.76 \\
en CAT (TATA-less) & GEM3 & 1.0 & 1.0 \\
& GEM en & 0.62 & 0.66 \\
& GEM $h s p$ & 0.67 & 0.51 \\
& GEM MET- & & \\
& TATA & 0.66 & 0.54 \\
\hline
\end{tabular}

Competition experiments were performed with the indicated reporter plasmids and competitor plasmids as described in $\mathrm{Ma}$ terials and methods. Transfections contained either $4 \mu \mathrm{g}$ of ActPPA or $4 \mu \mathrm{g}$ of Act-TFIID, as indicated at the top. To determine the effect of Act-TFIID on promoter activity during competition, CAT activities were normalized so that the values from cotransfections with GEM3 and Act-PPA were set at 1.0. 
TFIID was added during competition between the en CAT reporter and the two TATA-containing competitor plasmids, no increase in expression from the en promoter was detected. Instead, a further decrease in CAT activity was observed consistently with both (Table 3 ). These results indicate that increased expression of TFIID alone cannot restore activity from a TATA-less promoter during competition with TATA-containing for TATAlacking/ promoter fragments, suggesting that another, unidentified protein that can associate with both kinds of promoters is limiting for TATA-lacking promoters. The further decrease brought about by the TATA-containing competitor plasmids in the presence of excess TFIID suggests that TFIID can increase the association of this factor with the competing plasmid.

\section{Comparison of activated expression from minimal TATA-containing and TATA-less promoters in the presence of higher levels of TFIID reveals opposite effects}

The results from the competition experiments suggest that a TFIID-associated factor(s) may be limiting for expression from the TATA-less promoters tested. These findings are consistent with previous in vitro studies demonstrating that transcription from a TATA-less promoter composed of the initiator elements from the murine terminal deoxynucleotidyltransferase (TdT) gene or the adenovirus major late (AdML) promoter (Smale and Baltimore 1989) and the GC boxes constituting Splbinding sites from the SV40 early promoter (Dynan and Tjian 1983) requires the human TFIID fraction, but is not supported by purified yeast TFIID or recombinant hTFIID (Pugh and Tjian 1990; Smale et al. 1990). We therefore wanted to test the effect of increased TFIID levels on expression from this promoter in vivo to compare the results with those obtained with a similar Spl-responsive, TATA-containing promoter. In addition, with such synthetic constructs the effects of "promoter strength" on TFIID responsiveness can be assayed systematically simply by altering the concentration of $\mathrm{Spl}$ expression vector included in the transfections.

We first constructed reporter plasmids containing the GC boxes alone (G CAT) or in combination with either the AdML promoter TATA box (GT CAT) or the TdT initiator (GI CAT) inserted upstream of the CAT gene. Comparison of the CAT activities obtained from these reporters in the presence of $\mathrm{Spl}$ (produced from Act $\mathrm{Spl}$ ) confirmed that the initiator element was recognized as a functional element in Drosophila cells, as its removal caused a significant decrease (10- to 30-fold) in Sp l-mediated activation (data not shown). As was the case with other TATA-less promoters tested, the weak basal expression (i.e., in the absence of Spl) from GI CAT was not enhanced by increased TFIID expression. In contrast, expression from GT CAT was activated $\sim 20$-fold under the same conditions (data not shown). Increasing concentrations of Act Spl resulted in strong activation of expression from both GI CAT and GT CAT, but overexpression of TFIID with Spl had strikingly different ef- fects on the activation levels observed. Activity from the TATA-containing reporter GT CAT was further activated 4- to 14-fold by overexpression of TFIID at all Act Spl concentrations tested. (Note that some of this increase may reflect elevated $\mathrm{Spl}$ concentrations, as the exogenous TFIID may have increased its expression. However, as shown in Table 1, activation of the strong Actin 5C promoter by TFIID overexpression was at most twofold, which is insufficient to account for the GT activation levels observed.) These findings are entirely consistent with the results obtained with natural TATAcontaining promoters described above, including the observation that the lower the promoter activity is, the greater the response to TFIID. In contrast, expression from the TATA-less reporter GI CAT was inhibited (by a factor of $\sim 3$ ) at all Act Spl concentrations tested. These findings are also consistent with our observations with natural TATA-lacking promoters, although the inhibitory effect was significantly greater, perhaps reflecting the minimal nature of the synthetic GI CAT promoter. In addition, the approximately threefold reductions observed may well underestimate the actual inhibition if, as mentioned above, TFIID overexpression enhances Sp1 expression somewhat. Together, these results provide strong support for the notion that TFIID can be limiting for expression in vivo from TATA-containing, but not TATA-lacking, promoters. However, TFIID is able to functionally interact with a factor required for TATAless promoter expression.

\section{Discussion}

The isolation of a cDNA encoding Drosophila TFIID (Hoey et al. 1990; see also Muhich et al. 1990) allowed us to create expression plasmids encoding full-length and truncated forms of this protein. We have shown that wild-type and mutant TFIID can be produced in a concentration-dependent manner from plasmids transfected into cultured Drosophila cells and that overexpression of the full-length protein can enhance expression dramatically from a weak minimal TATA-containing promoter. Expression from several different TATA-containing promoters was also activated by increased synthesis of TFIID, and the level of activation observed was inversely proportional to the strength of the promoter. Activity from TATA-less promoters, however, was not enhanced and, in some cases, was inhibited by increased expression of TFIID. In addition, competition experiments showed that the presence of excess TATA sequences could cause reductions in expression from TATA-containing, as well as TATA-lacking, promoters. Increased expression of TFIID restored activity from the TATAcontaining, but not the TATA-less, promoter during such competition.

\section{Overexpression of TFIID can increase basal transcription}

The enhanced expression from TATA-containing promoters brought about by the production of exogenous 

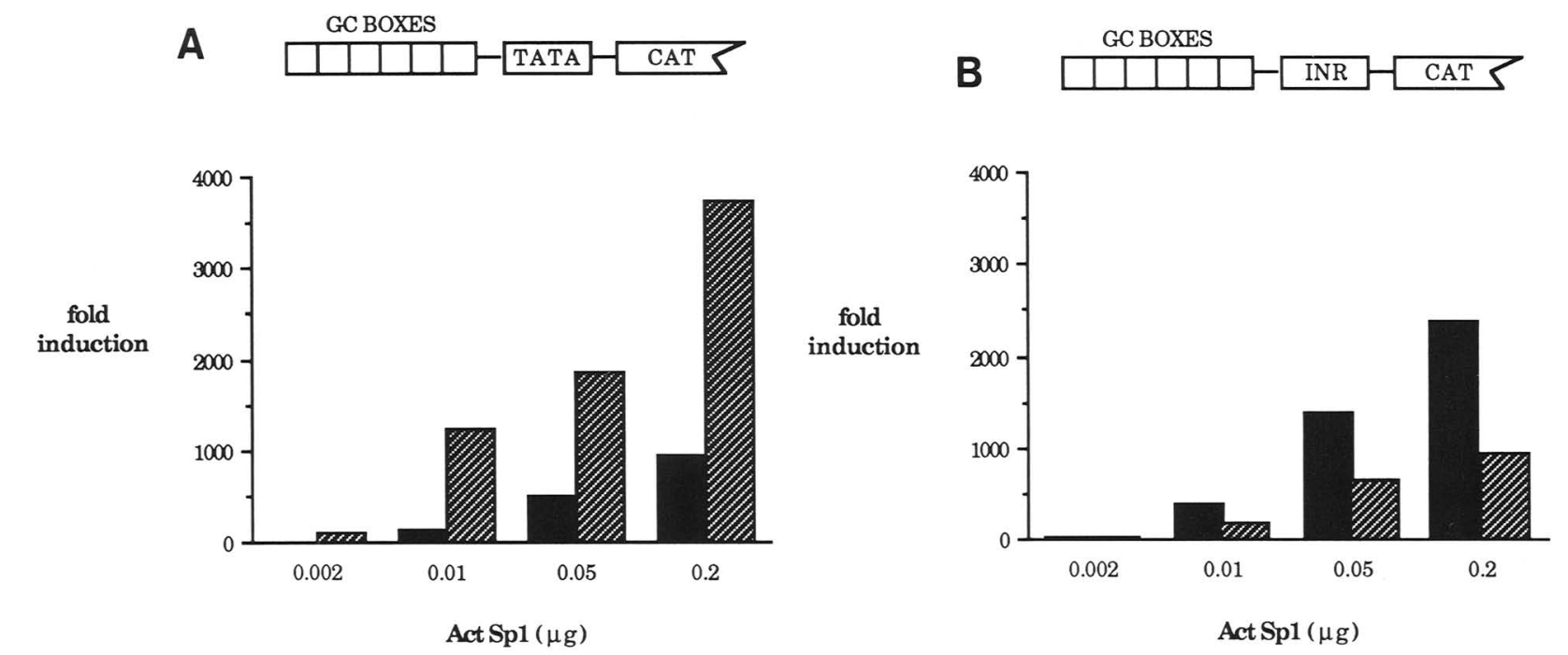

Figure 3. Overexpression of TFIID has opposite effects on the Spl-mediated activation of minimal TATA-containing and TATA-less promoters. (Solid bar) Act pBR; (hatched bar) Act flu-TFIID. (A) Bar graph showing the CAT activity observed from transfections including the TATA-containing reporter plasmid GT CAT, which is represented schematically above the plot, the indicated amount of $\mathrm{Spl}$ expression vector, and $4 \mu \mathrm{g}$ of either Act-PPA or Act flu-TFIID plus additional Act-PPA as necessary to bring the total amount of expression vector added to $4.6 \mu \mathrm{g}$. Transfections were performed as described in Materials and methods, except that each precipitate containing a total of $4.6 \mu \mathrm{g}$ of expression vector. $(B)$ Bar graph showing the CAT activity observed from transfections containing the TATA-less reporter plasmid GI CAT, which is represented schematically above the plot, the indicated amount of Spl expression vector, and $4 \mu \mathrm{g}$ of either Act-PPA or Act flu-TFID plus additional Act-PPA as necessary to bring the total amount of expression vector added to $4.6 \mu \mathrm{g}$. Transfections were performed as described in Materials and methods, except that each precipitate contained a total of $4.6 \mu \mathrm{g}$ of expression vector.

TFIID is a strong indication that the endogenous TFIID is maintained at lower levels in Schneider cells relative to other essential transcription factors. We found that expression increased as a function of TFIID concentration, demonstrating that the amount of functional TFIID present can be a factor that determines the transcription rate from a TATA-containing promoter. If a basal transcription factor other than TFIID was present in limiting concentrations, then increasing the TFIID concentration would have been without effect. Although it is conceivable that the increase in template concentration brought about by transfection significantly decreases the transcription factor-template ratio, our results nonetheless provide strong support for the notion that the available concentration of TFIID is significantly less than other essential transcription factors, which supports the view that factors that influence TFIID-TATA box interactions may play a key role in modulating promoter activity in vivo.

The increase in expression from a minimal TATAcontaining promoter was not the only effect that might have been predicted beforehand to arise from overexpression of TFIID. A possible alternative is that a decrease in TATA-containing promoter activity might have occurred by a mechanism analogous to the previously described phenomenon of squelching/Gill and Ptashne 1988). Squelching, or transcriptional interference (Meyer et al. 1989|, is an inhibition of transcription that can result from overexpression of a DNA-binding activator protein. Presumably, this reflects an interaction between the excess activator and a limiting essential transcription factor, resulting in sequestration of the factor in nonproductive complexes. Assuming that TFIID not bound to TATA sequences can interact stably with one or more other essential transcription factors, then the fact that overexpression of TFIID, or the DNA-binding defective mutant TFIID $\Delta \mathrm{Bgl}$, did not reduce expression from any promoter tested supports the view that TFIID itself, and not some other factor with which it interacts, is limiting for transcription from TATA-containing promoters.

It is conceivable that the level of functionally competent TFIID available within Schneider cells is controlled not by limiting its accumulation, but by maintaining TFIID in a conformation or complex that alone is incapable of associating with other essential transcription factors and/or binding productively to TATA sequences. This view is supported by the observation that TFIID characteristically behaves as a factor significantly larger than its molecular mass during purification (Reinberg et al. 1987; Pugh and Tjian 1990; Dynlacht et al. 1991) and is capable of associating with multiple factors that can inhibit basal transcription, and whose influence can be counteracted by the activator proteins USF (upstream stimulating factor) and Spl (Meisternerst et al. 1991; Meisternerst and Roeder 1991). The TFIID-mediated activation of basal transcription observed from minimal TATA-containing promoters may therefore be the result 
of an imbalance in the stoichiometry between such negatively acting factors and TFIID brought about by the overexpression of TFIID.

\section{Response to increased TFIID expression is inversely proportional to promoter strength}

It has long been proposed that some upstream activator proteins function during transcription complex formation to facilitate TFIID-TATA box interactions, and several in vitro studies have provided support for this idea. Binding experiments have shown that certain factors such as USF or GAL4 can bind cooperatively with TFIID (Sawadogo and Roeder 1985; Sawadogo 1988), alter its DNase I footprint (Horikoshi et al. 1988a,b), and/or facilitate binding of TFIID during competition with nucleosome assembly (Workman et al. 1988, 1990, 1991; Meisternerst et al. 1990). Several classes of transcriptional activators appear to function through TFIID using a class of intermediary molecules known as coactivators or adaptors (Berger et al. 1990; Kelleher et al. 1990; Martin et al. 1990; Pugh and Tjian 1990), some of which have been partially purified (Dynlacht et al. 1991; Meisternerst et al. 1991). Intriguingly, the activation domains of the viral proteins VP16 and Ela are capable of binding directly to TFIID (Stringer et al. 1990; Horikoshi et al. 1991; Ingles et al. 1991; Lee et al. 1991). Thus, a number of activator proteins appear to affect, directly or indirectly, the rate and/or stability of the TFIID binding.

If the interaction of TFIID with the TATA box is a factor that influences the rate of transcription, then strong expression from a TATA-containing promoter must represent, to some degree, the ability of the promoter to recruit TFIID. Our experiments examining the effect of TFIID accumulation on promoters of different strength extend these in vitro studies by providing evidence that activator proteins can influence the ability of a promoter to recruit TFIID. The strong $h s p 70$ promoter fragment $(-37$ to +90$)$ that was only slightly affected by overexpression of TFIID contains binding sites presumably recognized by activator proteins (Parker and Topol 1984; Gilmour et al. 1989, 1990). Removal of some of

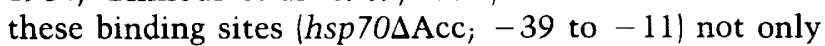
caused substantially decreased promoter activity but also increased the sensitivity of the promoter to the level of TFIID. Likewise, the reduced enhancement of expression by exogenous TFIID from the synthetic GT promoter observed at elevated Spl concentrations suggests that TFIID may be recruited more efficiently to the promoter under these conditions. These results also suggest that at least some of the TFIID-associated factors apparently required for Spl-mediated activation (Dynlacht et al. 1991; Meisternerst et al. 1991) must not be present in limiting concentrations or the exogenously expressed TFIID presumably would have been unable to function.

\section{Overexpression of TFIID can reduce expression} from TATA-less promoters

Because TFIID can function to initiate transcription upon binding to sequences that are divergent from the
TATAAA consensus (Nakajima et al. 1988; Hahn et al. 1989a; Singer et al. 1990; Wobbe and Struhl 1990) or to TATA sequences located downstream of the transcription start site (Carcamo et al. 1990), it is difficult to determine whether a given promoter truly lacks a functional TATA box. Mammalian promoters considered to be TATA-less commonly contain GC-rich sequences upstream of the start site that can be bound by Spl (Dynan et al. 1986; Swick et al. 1989) and by another mammalian factor, known as ETF, which activates expression only from promoters lacking TATA boxes (Kageyama et al. 1989). In yeast, a binding site for the transcriptional activator GCN4, which appears to interact specifically with yeast RNA polymerase II (Brandl and Struhl 1989), can substitute for a TATA element in a naturally occurring, or hybrid, promoter (Zalkin et al. 1984; Chen and Struhl 1989). Together, these results suggest that TATAless promoters may use a mechanism of initiation that does not require TFIID or one that uses TFIID in a manner unique to these promoters. Consistent with this latter proposal, results from reconstitution experiments have suggested that transcription in vitro from a synthetic TATA-less promoter requires a protein complex containing human TFIID associated with (an) auxiliary protein, designated the tethering factor, in addition to the other basal transcription factors and Spl (Pugh and Tjian 1990, 1991; Smale et al. 1990).

Our experiments have shown that expression from TATA-less promoters, unlike all TATA-containing promoters tested, cannot be increased by overexpression of TFIID, which suggests that TFIID is not limiting for TATA-lacking promoters. The observed inhibition of TATA-less promoter activity in vivo by TATA-containing fragments and/or overexpression of TFIID suggests that a factor able to interact with TFIID is limiting for TATA-less promoter expression. This was demonstrated most clearly in our experiments where overexpression of TFIID resulted in substantial reductions of Sp 1 -mediated activation of the synthetic TATA-lacking promoter GI. The simplest explanation for these findings is that overexpressed TFIID can squelch (Gill and Ptashne 1988) expression from the TATA-lacking promoters by sequestering a limiting factor. Therefore, the existence of limiting amounts of a factor(s), the function of which involves interaction with TFIID, could make TFIID de facto limiting for expression from TATA-less, as well as TATA-containing, promoters.

\section{Materials and methods}

\section{Recombinant plasmids}

All expression vectors were derivatives of the plasmid ActPPA, which contains the Drosophila actin 5C promoter and poly(A) site inserted into pBR322, as described in Han et al. (1989). Act-TFIID was created with the plasmid pFX29-6 (provided by T. Hoey and R. Tiian), which contains a 1.4-kb Drosophila TFIID (dTFIID) cDNA fragment inserted into the polylinker of Bluescript SK. This plasmid was cut with HphI, and the 3 ' overhang was removed by digestion with T4 DNA poly- 
merase and then digested with BamHI. The resulting $1.2-\mathrm{kb}$ fragment was inserted into Act-PPA that had been cut with $B a m H I$, filled in with Klenow, and digested with BgIII. A precursor to Act flu-TFIID $\Delta \mathrm{Bgl}$, known as Act-TFIID $\Delta \mathrm{Bgl}$, was also constructed from pFX29-6 by isolating a 1-kb fragment generated by cutting with $H p h \mathrm{I}$, removing the $3^{\prime}$ ends with $\mathrm{T} 4$ DNA polymerase, and digesting with BglII followed by fill-in with Klenow fragment. This $1-\mathrm{kb}$ fragment was then inserted into Act-PPA that had been digested with BamHI, filled in with Klenow and digested with EcoRV. Act P-flu (provided by K. Han), used to create the flu epitope-TFID fusion expression vectors, was constructed by cutting Act-PPA with $B a m \mathrm{HI}$ and then partially with SalI and ligating the isolated $6.2-\mathrm{kb} B a m H I-$ Sall fragment with annealed complementary synthetic oligonucleotides encoding a 10-amino-acid peptide from influenza virus (Field et al. 1988). Actin flu-TFIID was created using pFX-NdeLo (provided by T. Hoey and R. Tjian), which contains the dTFIID cDNA with an NdeI site introduced at nucleotide 183 of the cDNA. This plasmid was cut with Ndel, filled in with Klenow, and then digested with BstXI, which generated an 840-bp fragment that was ligated with a 350-bp HincII-NcoI fragment from Act P-flu and a 7-kb Nco-BstXI fragment from Act-TFIID. Actin flu-TFIID $\Delta$ Nde was constructed in the same way, except a 363-bp NdeI-Klenow-BstXI fragment from the plasmid pFXNde-SH (provided by T. Hoey and R. Tiian), which contains an NdeI site introduced at nucleotide 660 of the TFIID cDNA, was used in place of the 840-bp fragment from pFX-Nde-Lo. Act flu-TFIID $\Delta \mathrm{Bgl}$ was constructed by ligating the $1.2 \mathrm{~kb}$ NcoIBstXI fragment from Act flu-TFIID with the 7.1-kb NcoI-Bst XI fragment from Act-TFIID $\Delta \mathrm{Bgl}$. Act Spl (gift of T. Hoey and R. Tjian) is described in Courey and Tjian (1988).

All reporter plasmids except $U b x$ CAT, GT CAT, and GI CAT were constructed with the plasmid pUC-CAT, which is a derivative of pSVOCAT (Gorman et al. 1982). The plasmid Elb CAT (provided by K. Martin and M. Green; see Martin et al. 1990) contains a 17-bp oligonucleotide encoding the E 1b-TATA element inserted immediately upstream of the CAT gene and was activated to a maximum of $\sim 60$-fold when cotransfected with Act-TFIID. When the TATA-containing oligonucleotide was removed from Elb CAT by digestion with $\mathrm{XbaI}$ and $\mathrm{BamHI}$, followed by fill-in with Klenow and religation, the resulting plasmid, E1b $\Delta$ CAT, was also activated $(\sim 35$-fold) by cotransfection with Act-TFID. This response was presumably mediated by a cryptic TATA element located $\sim 80$ bp away from the $5^{\prime}$ end of the CAT that was encoded in the SP72 vector (Promega) used to construct the original plasmid. Analysis of the sequence and construction of pUC-CAT showed that no such cryptic TATA elements had been placed near the $5^{\prime}$ end of the CAT gene in this plasmid; therefore, the 570-bp Ncol-HindIII fragment that included the Elb-TATA element was removed from E1b CAT and inserted into pUC-CAT that had been digested with $\mathrm{NcOI}$ and HindIII, generating the Elb TATA CAT plasmid used in this study. The plasmid ElbSTATA CAT was created in a similar manner by inserting the 570 -bp NcoIHindIII fragment from E1b $\Delta$ CAT into pUC-CAT that been digested with $\mathrm{NcOl}$ and HindIII and showed no response to increasing concentrations of TFIID. Act5C CAT contains the 2.6$\mathrm{kb}$ promoter fragment from Act-PPA inserted into pUC-CAT. hsp70 CAT contains a 137-bp NruI-Pst fragment from the Drosphila 70K heat shock gene (Ingolia et al. 1980) inserted into pUC-CAT, whereas Hsp70 4 Acc contains a 43-bp MnlI-AccI fragment from the same promoter inserted into pUC-CAT. The plasmids Met-TATA CAT and en CAT (containing the en promoter fragment from bases -39 to +172 ) have been described in Han et al. (1989); the construction of SV2 CAT (SV40 E) is detailed in Gorman et al. (1982). The reporter ensHinc CAT was created by removing the 665 -bp HincII-Ncol fragment from en CAT and inserting it into ElbSTATA CAT that had been digested with SmaI and NcoI. copia CAT was constructed by inserting the 240-bp HindIII-XbaI fragment from the copialacZ internal control plasmid (Han et al. 1989) into HindIII and $X b a I$-digested pUC-CAT. Ubx CAT contains a promoter fragment from the Drosophila homeotic gene $U b x$ (Hogness et al. 1985) inserted into the plasmid vector pC4 CAT (Thummel et al. 1988). GI CAT (provided by T. Hoey) contains the SV40 GC boxes inserted 42 bp upstream of the murine TdT initiator sequence (Smale and Baltimore 1989), which were taken from the plasmid pSP42 Inr (Smale et al. 1990) and inserted into the CAT vector pBLCAT3 $\triangle H N$ (Luckow and Schutz 1987). A similar plasmid, known as GTI CAT (provided by T. Hoey), contains the SV40 GC boxes, the AdML promoter TATA sequence, and the TdT initiator sequences (all from plasmid "VII" as described in Smale et al. 1990) inserted upstream of the CAT gene in pBL3CATAHN. GT CAT was constructed by removing a NdeISacI fragment containing the GC boxes and AdML TATA box from GTI CAT and reinserting it into pBL3CAT $\triangle H N$.

All competitor plasmids contained promoter fragments inserted into the polylinker of the plasmid vector GEM3 (Promega). GEM hsp was constructed by removing the 400 -bp HindIII-EcoRI fragment (which contained 137 bp of $h s p 70$ promoter sequence and the first 246 bp of the CAT gene) from $h s p 70$ CAT reporter plasmid and inserting it into GEM3 that had been digested with HindIII and EcoRI. GEM hsp $\Delta$ TATA was constructed by digesting GEM hsp with $A c c I$, filling in with Klenow, and then religating. This removed a $\sim 40$-bp fragment from GEM $h s p$ that encodes the $h s p 70$ TATA box and surrounding sequences (bases -47 to -6 ). GEM en was constructed by ligating the 475-bp HindIII-EcoRI fragment /which contained $221 \mathrm{bp}$ of en promoter sequence and the first $246 \mathrm{bp}$ of the CAT gene) from en CAT with GEM3 digested with HindIII and EcoRI. GEM Met-TATA consisted of the 40-bp SalI-MscI fragment from Met-TATA CAT inserted into GEM3 that had been digested with Sall and SmaI.

\section{DNA transfection and transient expression assays}

Transient cotransfection assays were performed essentially as described in Han et al. (1989). Drosophila Schneider L2 cells were maintained at $24^{\circ} \mathrm{C}$ in M3 media supplemented with $10 \%$ fetal bovine serum (GIBCO) and were plated at $2-4 \times 10^{6}$ cells per $60-\mathrm{mm}$ tissue culture dish one day before transfection, and plasmid DNA was transfected with calcium phosphate coprecipitation. Except for the competition experiments or unless indicated otherwise, each precipitate contained $0.2-4 \mu \mathrm{g}$ of the appropriate TFIID expression vector, $2 \mu \mathrm{g}$ of reporter plasmid, 2 $\mu \mathrm{g}$ of copia-lacZ internal control plasmid, and GEM3 as needed to reach a total of $10 \mu \mathrm{g}$. For competition experiments, each precipitate contained $4 \mu \mathrm{g}$ of Act-PPA or Act-TFIID, $0.2 \mu \mathrm{g}$ of reporter plasmid, $2 \mu \mathrm{g}$ of copia-lacZ, and $5 \mu \mathrm{g}$ of GEM3 or the indicated competitor plasmid for a total of $11.2 \mu \mathrm{g}$. In all experiments, the total amount of actin 5C promoter in each sample was kept constant by the addition of Act-PPA as required. All experiments were repeated at least three times and many (e.g., the competitions/ up to six times. Transfected cells were incubated at $24^{\circ} \mathrm{C}$ for $48-50 \mathrm{hr}$, removed from tissue culture dishes by agitation, washed twice with PBS, resuspended in $0.1 \mathrm{ml}$ of $0.25 \mathrm{M}$ Tris- $\mathrm{HCl}(\mathrm{pH} 8.0)$, and frozen at $-70^{\circ} \mathrm{C}$. Whole-cell extracts were prepared by thawing and then sonicating for $2 \mathrm{~min}$ in an ultrasonic sonifier (Branson), followed by centrifugation at full speed for $10 \mathrm{~min}$ in a microcentrifuge. Transfection efficiencies were determined by assaying for $\beta$-galactosidase activ- 
ity as described in Han et al. (1989). Variations in transfection efficiencies within a given experiment were almost always less than twofold. CAT assays for promoter activity were also performed as described in Han et al. (1989).

\section{Western blot analysis}

For preparation of whole-cell extracts for Western blot analysis, Schneider cells were transfected and harvested exactly as described above, except that the cell pellet was resuspended in 1 $\mathrm{ml}$ of PBS instead of $0.25 \mathrm{M}$ Tris- $\mathrm{HCl}(\mathrm{pH} 8.0)$. A $0.25-\mathrm{ml}$ aliquot was removed from each resuspended sample and the cells pelleted, resuspended in $30 \mu \mathrm{l}$ of $0.25 \mathrm{M}$ Tris- $\mathrm{HCl}(\mathrm{pH} 8.0)$, and frozen at $-70^{\circ} \mathrm{C}$. Extracts were then prepared from these aliquots as described above and assayed for $\beta$-galactosidase activity. The remaining cells were pelleted, and the packed cell volume (PCV) for each sample was determined. (Cell pellets were resuspended in $1 \times \mathrm{PCV}$ of PBS plus $2 \times \mathrm{PCV}$ Laemmli loading buffer, and the samples were boiled for $5 \mathrm{~min}$ and centrifuged for $5 \mathrm{~min}$ in a microcentrifuge.) Aliquots were taken from each, with the amount removed determined by the $\beta$-galactosidase activity observed for the sample, and the volumes of the samples were made equal by the addition as needed of identically prepared extracts from mock-transfected cells. The proteins in these samples were resolved on a $10 \%$ SDS-polyacrylamide gel, transferred to nitrocellulose, and blotted with the mouse monoclonal antibody 12CA5 (Berkeley Antibody Company; Niman et al. 1983). A goat anti-mouse IgG antibody, conjugated to alkaline phosphate (Sigma Chemical Company), was used as the second antibody and was detected essentially as described in Harlow and Lane (1988).

\section{Acknowledgments}

We are grateful to M. Green, B. Johnson, K. Han, T. Hoey, K. Martin, D. Read, and R. Tjian for providing plasmids used in this study and to T. Hoey, B.F. Pugh, and R. Tjian for discussing their results prior to publication. We thank $M$. Wang for excellent technical assistance and K. Han, D. Read, L. Ryner, J. Wu, and $C$. Yan for technical advice and useful discussions. This work was supported by National Institutes of Health grant GM 37971 , and J.C. was supported by a predoctoral training grant from the National Institutes of Health.

The publication costs of this article were defrayed in part by payment of page charges. This article must therefore be hereby marked "advertisement" in accordance with 18 USC section 1734 solely to indicate this fact.

\section{References}

Abamyr, S.M., J.L. Workman, and R.G. Roeder. 1988. The pseudorabies immediate early protein stimulates in vitro transcription by facilitating TFIID : promoter interactions. Genes \& Dev. 2: 542-553.

Berger, S.L., W.D. Cress, A. Cress, S.J. Triezenberg, and L. Guarente. 1990. Selective inhibition of activated but not basal transcription by the acidic activation domain of VP16: Evidence for transcriptional adaptors. Cell 61: 1199-1208.

Brandl, C. and K. Struhl. 1989. Yeast GCN4 transcriptional activator protein interacts with RNA polymerase II in vitro. Proc. Natl. Acad. Sci. 86: 2652-2656.

Breathnach, R. and P. Chambon. 1981. Organization and expression of eucaryotic split genes coding for proteins. Annu. Rev. Biochem. 50: 349-383.

Bunch, T.A., Y. Grinblat, and L.S.B. Goldstein. 1988. Charac- terization and use of the Drosophila metallothionein promoter in Drosophila melanogaster cells. Nucleic Acids Res. 16: 1043-1061.

Buratowski, S., S. Hahn, P.A. Sharp, and L. Guarente. 1988. Function of a yeast TATA element-binding protein in a mammalian transcription system. Nature 334: 37-42.

Buratowski, S., S. Hahn, L. Guarente, and P.A. Sharp. 1989. Five intermediate complexes in transcription initiation by RNA polymerase II. Cell 56: $549-561$.

Carcamo, J., E. Maldonado, P. Cortes, M.-H. Ahn, I.-Ha, Y. Kasai, J. Flint, and D. Reinberg. 1990. A TATA-like sequence located downstream of the transcription initiation site is required for expression of an RNA polymerase II-transcribed gene. Genes \& Dev. 4: 1611-1622.

Carcamo, J., L. Buckbinder, and D. Reinberg. 1991. The initiator directs the assembly of a transcription factor IID-dependent complex. Proc. Natl. Acad. Sci. 88: 8052-8056.

Cavallini, B., J. Huet, J.-L. Plassat, A. Sentenac, J.-M. Egly, and P. Chambon. 1988. A yeast activity can substitute for the HeLa cell TATA box factor. Nature 334: 77-80.

Cavallini, B., I. Faus, H. Matthes, J.M. Chipoulet, B. Winsor, J.M. Egly, and P. Chambon. 1989. Cloning of the gene encoding the yeast protein BTF1Y, which can substitute for the human TATA box-binding factor. Proc. Natl. Acad. Sci. 86: 9803-9807.

Chen, W. and K. Struhl. 1989. Yeast upstream activator GCN4 can stimulate transcription when its binding site replaces the TATA element. EMBO /. 8: 261-268.

Cormack, B., M. Strubin, A.S. Ponticelli, and K. Struhl. 1991. Functional differences between yeast and human TFIID are localized to the highly conserved region. Cell 65: 341-348.

Courey, A.J. and R. Tjian. 1988. Analysis of $\mathrm{Spl}$ in vivo reveals multiple transcriptional domains, including a novel glutamine-rich motif. Cell 55: 887-898.

Davison, B.L., J.-M. Egly, E.R. Mulvihill, and P. Chambon. 1983. Formation of stable preinitiation complexes between eukaryotic class B transcription factors and promoter sequences. Nature 301: 680-686.

Dynan, W.S. 1986. Promoters for housekeeping genes. Trends Genet. 2: 196-197.

Dynan, W.S. and R. Tjian. 1983. The promoter-specific transcription factor $\mathrm{Spl}$ binds upstream sequences in the SV40 early promoter. Cell 35: 79-87.

Dynan, W.S., S. Sazer, R. Tiian, and R.T. Schimke. 1986. Transcription factor Spl recognizes a DNA sequence in the mouse dihydrofolate reductase promoter. Nature 319: 246248.

Dynlacht, B.D., T. Hoey, and R. Tjian. 1991. Isolation of coactivators associated with the TATA-binding protein that mediate transcriptional activation. Cell 66: 563-575.

Eisenmann, D.M., C. Dollard, and F. Winston. 1989. SPT15, the gene encoding the yeast TATA binding factor TFIID, is required for normal transcription initiation in vivo. Cell 58: 1183-1191.

Field, J., J.-I. Nikawa, D. Broek, B. MacDonald, L. Rodgers, I.A. Wilson, R. Lerner, and M. Wigler. 1988. Purification of a $R A S$-responsive adenyl cyclase complex from Saccharomyces cerevisiae by use of an epitope addition method. Mol. Cell. Biol. 8: 2159-2165.

Fire, A., M. Samuels, and P.A. Sharp. 1984. Interactions between RNA polymerase II, factors and template leading to accurate transcription. J. Biol. Chem. 259: 2509-2516.

Flanagan, P.M., R.J.I. Kelleher, W.J. Feaver, N.F. Lue, J.W. LaPointe, and R.D. Kornberg. 1990. Resolution of factors required for the initiation of transcription by yeast RNA polymerase II. J. Biol. Chem. 265: 11105-11107. 
Flores, O., I. Ha, and D. Reinberg. 1990. Factors involved in specific transcription by mammalian RNA polymerase II: Purification and subunit composition of transcription factor IIF. I. Biol. Chem. 265: 5629-5634.

Gasch, A., A. Hoffman, M. Horikoshi, R.G. Roeder, and N.H. Chua. 1990. Arabidopsis thaliana contains two genes for TFIID. Nature 346: 390-394.

Gill, G. and M. Ptashne. 1988. Negative effect of the transcriptional activator GAL4. Nature 334: 721-724.

Gill, G. and R. Tjian. 1991. A highly conserved domain of TFIID displays species specificity in vivo. Cell 65: 333-340.

Gilmour, D.S., G.H. Thomas, and S.C.R. Elgin. 1989. Drosophila nuclear proteins bind to regions of alternating $C$ and $T$ residues in gene promoters. Science 245: 1487-1490.

1990. UV crosslinking identifies four polypeptides that require the TATA box to bind the Drosophila $h s p 70$ promoter. Mol. Cell. Biol. 10: 4243-4255.

Gorman, C.M., L.F. Moffat, and B.H. Howard. 1982. Recombinant genomes which express chloramphenicol acetyltransferase in mammalian cells. Mol. Cell. Biol. 2: 1044-1051.

Hahn, S., S. Buratowski, P.A. Sharp, and L. Guarente. 1989a. Yeast TATA-binding protein TFIID binds to TATA elements with both consensus and nonconsensus DNA sequences. Proc. Natl. Acad. Sci. 86: 5718-5722.

- $1989 \mathrm{~b}$. Isolation of the gene encoding the yeast TATA binding protein TFIID: A gene identical to the SPT15 suppressor of Ty element insertions. Cell 58: 1173-1181.

$-1989 \mathrm{c}$. Identification of a yeast protein homologous in function to the mammalian general transcription factor, TFIIA. EMBO I. 8: 3379-3382.

Han, K.H., M.S. Levine, and J.L. Manley. 1989. Synergistic activation and repression of transcription by Drosophila homeobox proteins. Cell 56: 573-583.

Harlow, E. and D. Lane. 1988. Antibodies: A laboratory man ual. Cold Spring Harbor Laboratory, Cold Spring Harbor, New York.

Hoey, T., B.D. Dynlacht, M.G. Peterson, B.F. Pugh, and R. Tjian. 1990. Isolation and characterization of the Drosophila gene encoding the TATA box binding protein, TFIID. Cell 61: 1179-1186.

Hoffman, A., E. Sinn, T. Yamamoto, J. Wang, A. Roy, M. Horikoshi, and R.G. Roeder. 1990. Highly conserved core domain and unique $\mathrm{N}$ terminus with presumptive regulatory motifs in a human TATA factor. Nature 346: 387-390.

Hogness, D.S., H.D. Lipshitz, P.A. Beachy, D.A. Peattie, R.B. Saint, M. Goldschmidt-Clermont, P.J. Harte, E.R. Gavis, and S.L. Helfand. 1985. Regulation and products of the $U b x$ domain of the bithorax complex. Cold Spring Harbor Symp. Quant. Biol. 50: 181-194.

Horikoshi, M., M.F. Carey, H. Kakidani, and R.G. Roeder. 1988a. Mechanism of action of a yeast activator: Direct effect of Gal4 derivatives on mammalian TFIID-promoter interactions. Cell 54: 665-669.

Horikoshi, M., T. Hai, Y.-S. Lin, M.R. Green, and R.G. Roeder. 1988b. Transcription factor ATF interacts with the TATA factor to facilitate establishment of a preinitiation complex. Cell 54: 1033-1042.

Horikoshi, M., C.W. Wang, H. Fujii, J.A. Cromlish, P.A. Weil, and R.G. Roeder. 1989. Cloning and structure of a yeast gene encoding a general transcription factor TFIID that binds to the TATA box. Nature 341: 299-303.

Horikoshi, M., T. Yamamoto, Y. Ohkuma, P.A. Weil, and R.G. Roeder. 1990. Analysis of structure-function relationships of yeast TATA box binding factor TFID. Cell 61: 1171-1178.

Horikoshi, M., K. Maguire, A. Kralli, E. Maldonado, D. Reinberg, and R. Weinmann. 1991. Direct interaction between adeno- virus E1A protein and the TATA box binding transcription factor IID. Proc. Natl. Acad. Sci. 88: 5124-5128.

Ingles, C.J., M. Shales, W.D. Cress, S. Treizenberg, and J. Greenblatt. 1991. Reduced binding of TFIID to transcriptionally compromised mutants of VP16. Nature 351: 588-590.

Ingolia, T.D., E.A. Craig, and B.J. McCarthy. 1980. Sequence of three copies of the gene for the major Drosophila heat shock induced protein and their flanking regions. Cell 21: 669-679.

Johnson, P.F. and S.L. McKnight. 1990 . Eukaryotic transcriptional regulatory proteins. Annu. Rev. Biochem. 58: 799839 .

Kageyama, R., G.T. Merlino, and I. Pastan. 1989. Nuclear factor ETF stimulates transcription from promoters without a TATA box. J. Biol. Chem. 264: 15508-15514.

Kao, C.C., P.M. Lieberman, M.C. Schmidt, Q. Zhou, R. Pei, and A.J. Berk. 1990. Cloning of a transcriptionally active human TATA binding factor. Science 248: 1646-1649.

Kelleher, R.J., P.M. Flanagan, and R.D. Kornberg. 1990. A novel mediator between activator proteins and the RNA polymerase II transcription apparatus. Cell 61: 1209-1215.

Lee, W.S., C.C. Kao, G.O. Bryant, X. Liu, and A.J. Berk. 1991. Adenovirus E1A activation domain binds the basic repeat in the TATA box transcription factor. Cell 67: 365-376.

Lewin, B. 1990. Commitment and activation at pol II promoters: A tail of protein-protein interactions. Cell 61: 11611164.

Luckow, B. and G. Shutz. 1987. CAT construction with multiple unique sites for the functional analysis of eukaryotic promoters and regulatory elements. Nucleic Acids Res. 15: 5490 .

Martin, K.J., J.W. Lillie, and M.R. Green. 1990. Evidence for interaction of different eukaryotic transcriptional activators with distinct cellular targets. Nature 346: 147-152.

Meisternerst, M. and R.G. Roeder. 1991. Family of proteins that interact with TFIID and regulate promoter activity. Cell 67: 557-567

Meisternerst, M., M. Horikoshi, and R.G. Roeder. 1990. Recombinant yeast TFIID, a general transcription factor, mediates activation by the gene-specific factor USF in a chromatin assembly. Proc. Natl. Acad. Sci. 87: 9153-9157.

Meisternerst, M., A.L. Roy, H.M. Lieu, and R.G. Roeder 1991. Activation of class II gene transcription by regulatory factors is potentiated by a novel activity. Cell 66: 981-993.

Mermelstein, F.H., O. Flores, and D. Reinberg. 1989. Initiation of transcription by RNA polymerase II. Biochim. Biophys. Acta. 1009: 1-10.

Meyer, M.-E., H. Gronemeyer, B. Turcotte, M.-T. Bocquel, D. Tasset, and P. Chambon. 1989. Steroid hormone receptors compete for factors that mediate their enhancer function. Cell 57: 433-442.

Mitchell, P.J. and R. Tjian. 1989. Transcriptional regulation in mammalian cells by sequence-specific DNA binding proteins. Science 245: 371-378.

Mount, S.M. and G.R. Rubin. 1985. Complete nucleotide sequence of the Drosophila transposable element copia: Homology between copia and retroviral proteins. Mol. Cell. Biol. 5: 1630-1638.

Muhich, M.L., C.T. Iida, M. Horikoshi, R.G. Roeder, and C.S. Parker. 1990. cDNA clone encoding the Drosophila transcription factor TFIID. Proc. Natl. Acad. Sci. 87: 9148-9152.

Nakajima, N., M. Horikoshi, and R.G. Roeder. 1988. Factors involved in specific transcription by mammalian RNA polymerase II: Purification, genetic specificity and TATA-boxpromoter interactions of TFIID. Mol. Cell. Biol. 8: 40284040.

Niman, H.L., R.A. Houghten, L.E. Walker, R.A. Reisfeld, I.A. 
Wilson, J.M. Hogle, and R.A. Lerner. 1983. Generation of protein-reactive antibodies by short peptides is an event of high frequency: Implications for the structural basis of immune recognition. Proc. Natl. Acad. Sci. 80: 4949-4953.

Ohkuma, Y., H. Sumimoto, M. Horikoshi, and R.G. Roeder. 1990. Factors involved in specific transcription by mammalian RNA polymerase II: Purification and characterization of general transcription factor TFIIE. Proc. Natl. Acad. Sci. 87: 9163-9167.

Parker, C.S. and J. Topol. 1984. A Drosophila RNA polymerase II transcription factor contains a promoter-specific-DNAbinding activity. Cell 37: 273-283.

Peterson, M.G., N. Tanese, B.F. Pugh, and R. Tjian. 1990. Functional domains and upstream activation properties of cloned human TATA binding protein. Science 248: 1625-1630.

Poole, S.J., L.M. Kauvar, B. Drees, and T. Kornberg. 1985. The engrailed locus of Drosophila: Structural analysis of an embryonic transcript. Cell 40: 37-43.

Ptashne, M. and A.A.F. Gann. 1990. Activators and targets. Nature 346: 329-331.

Pugh, B.F. and R. Tjian. 1990. Mechanism of transcriptional activation by $\mathrm{Spl}$ : Evidence for coactivators. Cell 61: 11871197.

- 1991. Transcription from a TATA-less promoter requires a multisubunit TFIID complex. Genes \& Dev. 5: 1935-1945.

Reddy, P. and S. Hahn. 1991. Dominant negative mutations in yeast TFIID define a bipartite DNA-binding region. Cell 65: 349-357.

Reinberg, D. and R.G. Roeder. 1987. Factors involved in specific transcription by mammalian RNA polymerase II: Purification and functional analysis of initiation factors IIB and IIE. I. Biol. Chem. 262: 3310-3321.

Reinberg, D., M. Horikoshi, and R.G. Roeder. 1987. Factors involved in specific transcription by mammalian RNA polymerase II: Functional analysis of initiation factors IIA and IID and identification of a new factor operating at sequences downstream of the initiation site. I. Biol. Chem. 262: 33223330.

Sawadogo, M. 1988. Multiple forms of the human gene-specific transcription factor USF: DNA binding properties and transcriptional activity of the purified HeLa USF. J. Biol. Chem. 263: 11994-12001.

Sawadogo, M. and R.G. Roeder. 1985. Interaction of a genespecific transcription factor with the adenovirus major late promoter upstream of the TATA box region. Cell 43: 165175.

Sawadogo, M. and A. Sentenac. 1990. RNA polymerase B (II) and general transcription factors. Annu. Rev. Biochem. 59: 711754.

Schmidt, M.C., C.C. Kao, R. Pei, and A.J. Berk. 1989. Yeast TATA-box transcription factor gene. Proc. Natl. Acad. Sci. 86: 7785-7789.

Soeller, W.C., S.J. Poole, and T. Kornberg. 1988. In vitro transcription of the Drosophila engrailed gene. Genes \& Dev. 2:68-81.

Singer, V.L., C.R. Wobbe, and K. Struhl. 1990. A wide variety of DNA sequences can functionally replace a yeast TATA element for transcriptional activation. Genes \& Dev. 4: 636645.

Smale, S. and D. Baltimore. 1989. The "initiator" as a transcription control element. Cell 57: 103-113.

Smale, S., M.C. Schmidt, A.J. Berk, and D. Baltimore. 1990. Transcriptional activation by $\mathrm{Spl}$ as directed through TATA or initiator: Specific requirement for mammalian transcription factor IID. Proc. Natl. Acad. Sci. 87: 4509-4513.
Stringer, K.F., C.J. Ingles, and J. Greenblatt. 1990. Direct and selectively binding of an acidic transcriptional activation domain to the TATA-box factor TFIID. Nature 345: 783-786.

Sumimoto, H., Y. Ohkuma, T. Yamamoto, M. Horikoshi, and R.G. Roeder. 1990. Factors involved in specific transcription by mammalian RNA polymerase II: Identification of general transcription factor TFIIG. Proc. Natl. Acad. Sci. 87: 91589162.

Swick, A.G., M.C. Blake, and J.W. Azizkhan. 1989. Functional analysis of GC element binding and transcription in the hamster dihydrofolate reductase gene promoter. Nucleic Acids Res. 17: 9291-9304.

Thummel, C.S., A.M. Boulet, and H.D. Lipshitz. 1988. Vectors for Drosophila p-element-mediated transformation and tissue culture transfection. Gene 74: 445-456.

Van Dyke, M.W., R.G. Roeder, and M. Sawadogo. 1988. Physical analysis of transcription preinitiation complex assembly on a class II gene promoter. Science 241: 1335-1338.

Wampler, S.L., C.T. Tyree, and J.T. Kadonaga. 1990. Fractionation of general RNA polymerase II transcription factors from Drosophila embryos. I. Biol. Chem. 265: 21223-21231.

Wilson, I.A., H.L. Niman, R.A. Houghten, A.R. Cherenson, M.L. Connolly, and R. Lerner. 1984. The structure of an antigenic determinant in a protein. Cell 37: 767-778.

Wobbe, C.R. and K. Struhl. 1990. Yeast and human TATA-binding proteins have nearly identical DNA sequence requirements for transcription in vitro. Mol. Cell. Biol. 10: 38593867.

Workman, J.L. and R.G. Roeder. 1987. Binding of transcription factor to the major late promoter during in vitro nucleosome assembly potentiates subsequent initiation by RNA polymerase II. Cell 55: 211-219.

Workman, J.L., S.M. Abamyr, W.A. Cromlish, and R.G. Roeder. 1988. Transcriptional regulation by the immediate early protein of pseudorabies virus during in vitro nucleosome assembly. Cell 55: 211-219.

Workman, J.L., R.G. Roeder, and R.E. Kingston. 1990. An upstream transcription factor, USF (MLTF) facilitates the formation of preinitiation complexes during in vitro chromatin assembly. EMBO 1. 9: 1299-1308.

Workman, J.L., I.C.A. Taylor, and R.E. Kingston. 1991. Activation domains of stably bound GAL4 derivatives alleviate repression of promoters by nucleosomes. Cell 64: 533-544.

Zalkin, H., J.L. Paluh, M. vanCleemput, W.S. Moye, and C. Yanofsky. 1984. Nucleotide sequence of Saccharomyces cerevisiae genes TRP2 and TRP3 encoding bifunctional anthranilate synthase: Indole-3-glycerol phosphate synthase. I. Biol. Chem. 259: 3985-3992. 


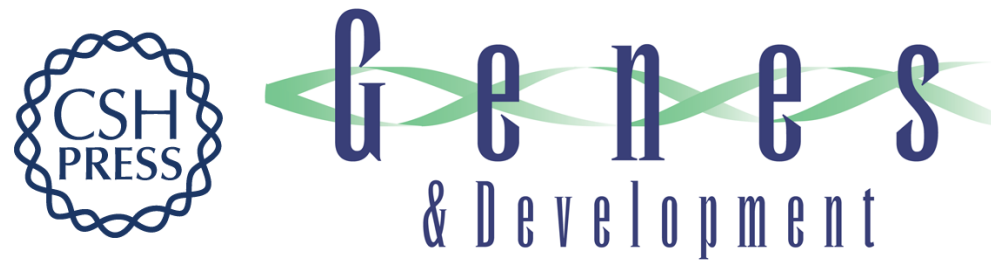

\section{TFIID can be rate limiting in vivo for TATA-containing, but not TATA-lacking, RNA polymerase II promoters.}

$\mathrm{J}$ Colgan and J L Manley

Genes Dev. 1992, 6:

Access the most recent version at doi:10.1101/gad.6.2.304

References This article cites 93 articles, 38 of which can be accessed free at:

http://genesdev.cshlp.org/content/6/2/304.full.html\#ref-list-1

License

Email Alerting

Service

Receive free email alerts when new articles cite this article - sign up in the box at the top right corner of the article or click here.

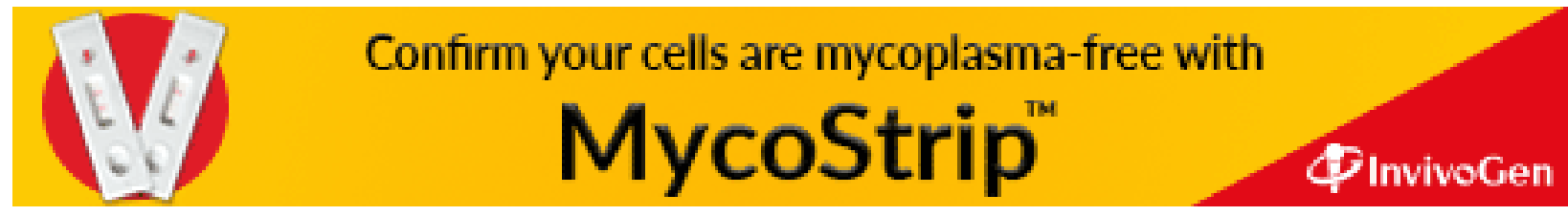

